\title{
A Proposed Scheme for Epidemic Routing with Active Curing for Opportunistic Networks
}

\author{
J.P. Tower and T.D.C. Little \\ Department of Electrical and Computer Engineering \\ Boston University, Boston, MA 02215 \\ \{jpt2002,tdcl\}@bu.edu
}

MCL Technical Report No. 01-01-2008

\begin{abstract}
Opportunistic networking is emerging as a technique to exploit chance encounters among mobile nodes, and is distinct from previously studied behaviors found in sensor and ad hoc networking research. In this paper, we explore contributions in epidemic data dissemination and mobile ad hoc networks applicable to opportunistic networking, and propose an extension to prior work based on active cures. Our scheme, called SERAC, increases the rate at which cure messages are propagated in a fragmented network for the purpose of reducing the overhead of outstanding yet incompletely disseminated messages. Preliminary analyses demonstrate the feasibility of performance gains under the opportunistic networking model.
\end{abstract}

Keywords -- Opportunistic networking (OPPNET), delay-tolerant networking (DTN), mobile ad hoc networking (MANET), epidemic routing (ER).

In Proc. $1^{\text {st }}$ IEEE Intl. Workshop on Opportunistic Networking, Okinawa, Japan, March 2008. This material is based upon work support by the National Science Foundation under Grant No. CNS0435353 . 


\section{Introduction}

The ubiquity of small, untethered devices emerging from a variety of related fields of sensor networking, cellular telephony, consumer electronics, vehicular communications, RFID, and mobile computing is enabling new and unexpected forms of networking. The idea of a few tens of nodes interacting in a closed, rapidly deployed mobile ad hoc network to support point-to-point communications today appears to be a narrow interpretation of possible applications of MANETs. Instead, many applications are more suitably described as delay-tolerant and reliant on opportunistic connectivity. Examples include inter-vehicle communication in VANETs [15][20], wildlife monitoring [16][17] activity monitoring in humans [18], and data mules [19] to name a few.

With the increasing popularity of mobile computing there is a need to develop routing techniques for mobile ad hoc networks (MANETs). Moreover, an extensive amount of research has resulted in several routing protocols (e.g., DODV [10], DSR [11], AODV [12], and derivatives) that function under the assumption of a generally connected network of nodes. However, in the case of a highly partitioned node topology or delay tolerant network (DTN) where individual nodes may be completely disconnected for large periods of time, and no end-to-end multi-hop path can be found from sender to receiver, these types of protocols can break down.

Furthermore, the emergence of wireless sensor networks (WSNs) for industrial, military, and ecological monitoring is due in large part to their low cost and the promise of network connectivity. These WSNs can be deployed in remote locations or areas of disabled network infrastructure if they possess MANET characteristics of adaptability. And, as the technology continues to evolve increasing sensory capabilities, processing power, storage space, and battery life, we envision mobile WSN robots that can perform exploration, object and event detection, and the exchange of critical information under a DTN scenario. These opportunistic networks (OPPNETs) [13] are expected to be applied in a variety of situations where fixed infrastructure is not feasible or unavailable.

We explore the intersection of delay tolerant schemes and epidemic routing through the design of a new scheme to propagate data in scenarios in which one primarily seeks completeness of data delivery while balancing timeliness and efficiency. The remainder of this paper is organized as follows: In Section 2, we discuss the directly relevant related work. In Section 3, we briefly outline two background techniques that are utilized as part of our system. Section 4 covers SERAC, our proposed epidemic routing scheme. Section 5 frames the foundation for the analysis needed to verify our system. Finally, Section 6 concludes the paper and summarizes future work.

\section{Related Work}

Models and analyses have been considered for epidemic information dissemination in data networks [8]; however, there are some gaps in adapting this work to the characteristics of MANETs or DTNs due to the assumption of network connectivity.

The use of Epidemic Routing (ER) in a delay tolerant wireless network appears in the often cited work of Vahdat and Becker [1]. Here, in a highly disconnected MANET, messages are forwarded randomly each time nodes establish contact in the hope of eventually reaching a receiver node. However, this work focuses on the bootstrap or startup phases of operation, and does not consider the details of resource consumption and possible means of achieving reclamation of resources. 
PRoPHET is proposed as an enhancement to the basic functionality of ER in which connectivity/position history of each node can be leveraged to make forwarding decisions, thus reducing traffic and increasing throughput with reduced latency [2].

The Island Hopping scheme [3] suggests that nodes often move in and out of areas with Concentration Points (CPs). CPs are characterized by regions in which many nodes are in contact with each other. Using a graph of the CPs, the authors propose to move data from CP to CP via node mobility. This scheme requires that nodes form groups of close proximity and that destination geographical locations are known a priori.

Wang et al. propose the use of Erasure Coding (EC)-based routing in opportunistic networks [4]. Chen et al. [5] extend EC with a hybrid technique. These methods decompose messages into smaller units with redundancy, permitting reconstruction of the original with a subset of the smaller units. The resulting decomposition is suitable for epidemic propagation with fixed and predictable overhead. In the hybrid version, there is an added feature to more aggressively forward those pieces by duplication and by using a more aggressive ER mechanism to move the second set of messages. But, the algorithms required to encode and decode the messages can be computationally expensive and unsuitable for a variety of platforms (e.g., energy limited). The authors allude to "healing" an infected network but do not explore this technique.

Finally, in an unpublished paper [6] and then later in Controlled Flooding [7], the concept of using acknowledgement (ACK) messages to passively cure an epidemic is proposed with the goal of reducing resource consumption by either piggy-backing the ACKs on other epidemics or via light-weight ACK messages that utilize the same mechanisms of the original infections. However, neither investigates the approach of actively using a "cure" to restrict infections and thus reduce resource utilization.

While many techniques have been suggested for optimizing the use of epidemic data forwarding in DTNs, these do not focus on how to best optimize the curing to ensure rapid annihilation of the infection without excessive added overhead. In this paper, we are exploring the novel contribution of Active Cures to epidemically-based routing schemes in opportunistic networks without the need for positional awareness or strong assumptions on the behavior of nodes within the system and with little computational or communication overhead added.

\section{Background}

As reviewed in the related work there has been a considerable effort applied to the use of epidemic message forwarding in DTNs. In the following subsections we elaborate on prior work that is used as part of our proposed routing mechanism. Our goal is to achieve data dissemination in a highly fragmented opportunistic network while maintaining limited resource consumption and satisfactory overall performance.

\subsection{Epidemic Routing}

In a highly fragmented network, one can propagate messages from sender node $(\mathrm{S})$ to receiver node $(\mathrm{R})$ via forwarding nodes (F) by using the point-to-point single-hop neighbor encounters that occur when two or more mobile nodes have a probabilistic encounter [1]. In the very basic scheme, when a sender node encounters either the receiver node or any other node, it will pass its message to an encountered node if it has not already been "infected" by that message. Thus, data propagates outwardly from a 
sender node in an "epidemic" fashion repetitively infecting additional nodes. The advantages of this scheme are: (1) there is no need for knowledge of the network topology or even node position (location) and (2) communication between disconnected nodes is possible. It has been shown that, with sufficient resources, message delivery is guaranteed in finite time [8].

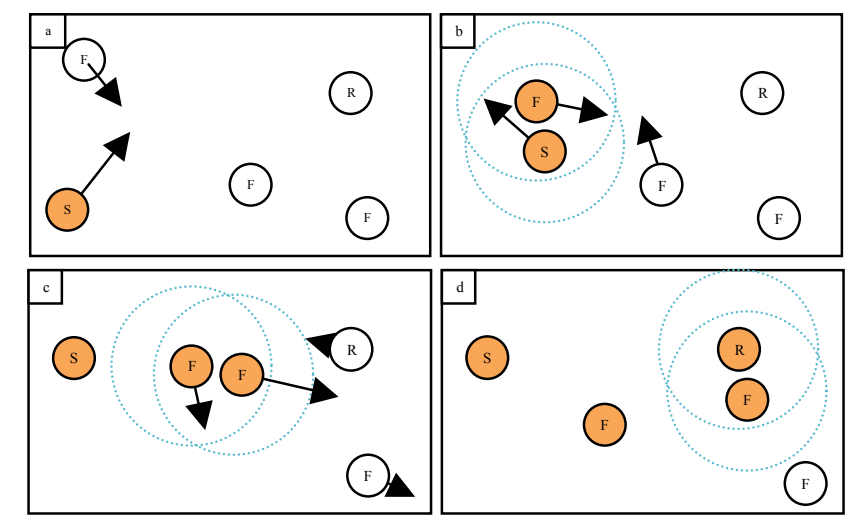

Figure 1: Sender node (S) produces a data message destined for Receiver node (R), which is randomly passed via Forwarding nodes (F); ultimately transfering a message from source to receiver [7].

Figure 1 illustrates the propagation of a message in epidemic routing. As nodes come into contact with each other, i.e. nodes arrive within radio range of one another (shown with dashed circles), the infection propagates from node to node. This is the basic mechanism by which messages are forwarded through a sparsely connected mobile ad hoc network of nodes using Epidemic Information Dissemination [8]. In the case of a message being sent from a single sender to 1 to $\mathrm{n}$ receivers there is little need to reclaim used resources; however, when extending the above scenario to many senders, many receivers, and over an extended time period, there may be hundreds or thousands of "independent" infections competing for the same underlying resources.

To further enhance the epidemic routing mechanism, we adopted the following parameters from Controlled Flooding [7] to allow for control over the rate and completeness with which an infection spreads through the network:

Beacon Interval (BI): In order to discover neighboring nodes within transmission range, each node must transmit a periodic HELLO Beacon. The BI is the time period between HELLO Beacon transmissions.

Times-to-Send (TTS): This is the maximum number of times that any node will forward a given message.

Retransmission Wait Time (RWT): After forwarding a message, a node will make the message dormant for a period of RWT before it re-transmits that same message.

\subsection{Message Exchange}

Once a pair of nodes establishes a connection in a chance rendezvous, they engage in an anti-entropy session (AES) in which the nodes update their messages and ACK buffers to mutually infect one another 
with the most valid data [6]. The basic steps in this process are to (1) exchange ACK messages (cures), (2) update both ACK and message buffers, and then (3) exchange data messages (infections). We illustrate the specific steps of this exchange below (Figure 2) and address the details in Section 4.

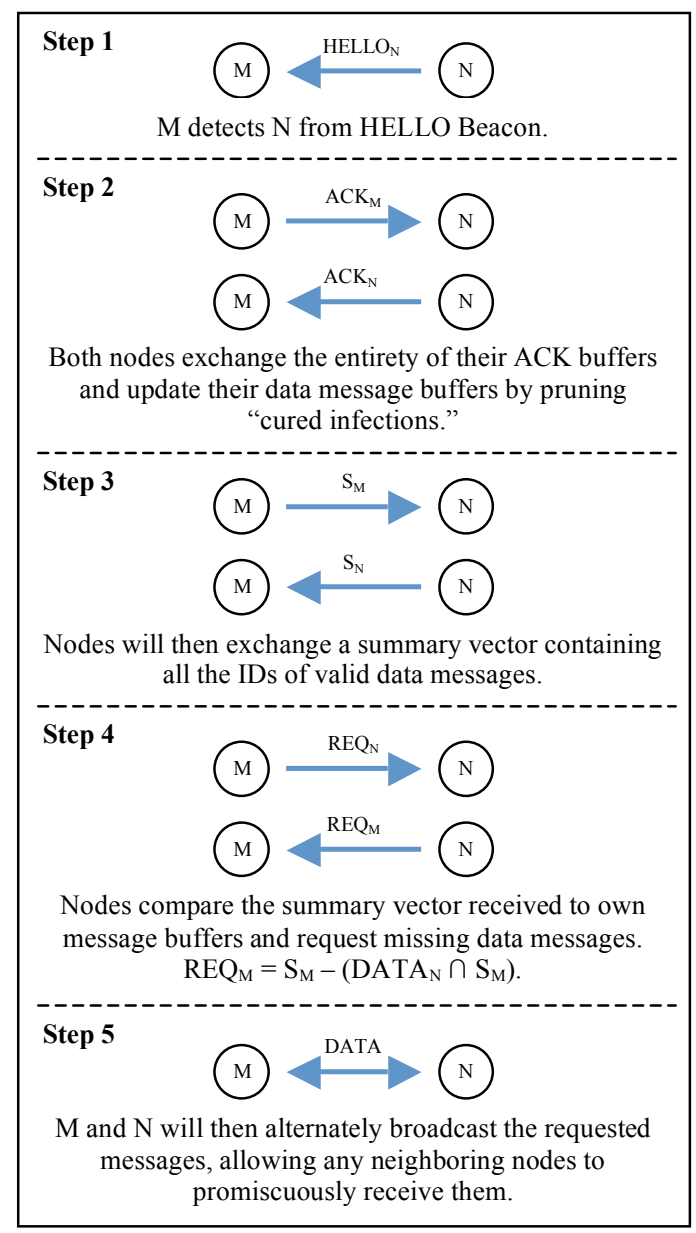

Figure 2: Shows the basic steps of an anti-entropy session (AES).

After the exchange concludes, both nodes will carry the same ACK buffer $\left(\mathrm{ACK}_{\text {buf }}=\mathrm{ACK}_{\mathrm{M}}+\mathrm{ACK} \mathrm{K}_{\mathrm{N}}\right)$. However, by enforcing a RWT on successfully forwarded messages, some messages in the buffer are labeled as dormant, and these messages are excluded from the summary vectors that are exchanged during an AES. Therefore, an AES may close without the same infections in each node, while both nodes will have a complete union of the original ACK buffers. This ensures that the cure can annihilate an infection by promoting the distribution of the cure. This process is described below in our extensions to the existing work. 


\section{SERAC Algorithm}

Our proposed Scheme for Epidemic Routing with Active Curing (SERAC) is based on Epidemic Routing [1] and techniques from Controlled Flooding [7]. Here we present the key novel contribution of our algorithm: Active Curing, which is founded in ACK messages (cures) being propagated in an epidemic fashion more aggressively than the original data messages (infection). In addition we outline several of the fundamental data structures and heuristics needed for the implementation of SERAC.

\subsection{Active Curing}

The controlled flooding scheme [7] shows that passive curing reduces overall message traffic and resource usage. This is demonstrated through simulation; however, in passive curing the ACK messages (cures) are propagated through the network in the same way as the original data messages (infection). In a very large network, of 1000's or possibly 10,0000's, an undirected infection, originating at the sender and expanding outward, will not be completely healed by a passive cure because the infection and cure move in the network at the same rate.

Therefore, in active curing we propose that the ACK messages are propagated through the network preferentially. Also, because the cure messages are substantially smaller (consisting of two node address for sender/receiver pair, each 4 bytes, and a 16-bit unsigned integer for message index - total 10 bytes needed) than the original infection messages (ranging from 100+ bytes, e.g. TXT messages, to 100's of $\mathrm{kB}$, e.g. picture files), nodes are able to buffer the cures for a greater amount of time at lower resource consumption to ensure that cured infections are completely annihilated. The details of the message structure and buffering heuristics are explained below.

\subsection{Additional Protocol Details}

The goal of active curing is for ACK messages to propagate faster, further, and more completely than the original data messages, while using fewer overall system resources (both communication and storage). Here we focus on (1) the information that is to be associated with each data message, (2) the heuristics of the data message buffer, (3) the structure of the ACK message buffer and how it is used, and (4) and the composition of the SERAC control messages themselves.

\subsubsection{SERAC Data Message Header}

Within the header of each data message we require a sender address $\left(A_{S}\right)$, a receiver address $\left(A_{R}\right)$, a message index $(M I)$ that is unique to $\left(A_{S}, A_{R}\right)$, and a Times-to-Send (TTS) value. The sender-receiverindex triple will uniquely identify that message and be used in the corresponding $\mathrm{ACK}$. By including a TTS value, each sender has some control over how the message propagates, i.e. controls "priority." The SERAC header, shown in Figure 3, adds only 12 bytes to the original message. 


\begin{tabular}{|c|c|}
\hline \multicolumn{2}{|c|}{ Sender Address $\left(\mathrm{A}_{\mathrm{S}}\right)-32$ bits (IPv4) } \\
\hline \multicolumn{2}{|c|}{ Destination Address $\left(\mathrm{A}_{\mathrm{D}}\right)-32$ bits (IPv4) } \\
\hline Message Index $(\mathrm{MI})-$ & Times-to-Send (TTS) \\
16 bits & -16 bits \\
\hline
\end{tabular}

Figure 3: SERAC Data Message header.

\subsubsection{Data Message Buffer}

In addition to the information in the SERAC header each message is stored with a sent timestamp, $t_{\text {sent }}$, which is used in conjunction with the Retransmission Wait Time (RWT), indicating whether the infection is active or dormant. Then buffer overflows are simply handled by discarding messages with a lower remaining-TTS count. This is a reasonable heuristic under the assumption that a message, which has been transmitted more often, will continue to be propagated through the network by other nodes.

\subsubsection{ACK Message Buffer}

ACK Message buffers are implemented using a hash table using a hash function based on the senderreceiver pair $\left(A_{S}, A_{R}\right)$. In each location where ACKs have been received, there is a list of begin $\left(M I_{\text {begin }}\right)$ and end $\left(M I_{\text {end }}\right)$ message-index pairs corresponding to a continuous series of MIs for received ACKs. As previously indicated, others have shown that messages will eventually reach all nodes in the system using epidemic routing [14]; therefore, by keeping only the beginning and ending indices of an unbroken series of ACKs, we can greatly reduce the storage (and transmission) costs of cures, thus allowing them to survive longer than the infections and ultimately approach the goal of reaching every node.

After updating its ACK buffer, node $M$ creates a Summary Vector $\left(S_{M}\right)$ by iterating through its Data Message buffer adding "uncured" data messages to $S_{M}$ and deleting the newly "cured" data messages. Thus, the design of the ACK buffer is such that a quick lookup based on the $\left(A_{S}, A_{R}, M I\right)$ triple is possible.

\subsubsection{SERAC Control Messages}

Finally, SERAC Control Messages are based on the compact structure of the ACK buffer. Each ACK message is comprised of a header followed by a variable length list of sender-receiver $\left(A_{S}, A_{R}\right)$ blocks. Each block has an index-pair count and a list of $M I_{\text {begin }}$ and $M I_{\text {end }}$ indices as illustrated in Figure 4. This highly compact message format is used to transmit ACK Buffer contents (ACK), Summary Vector (S), and the Request Vector (REQ) as described in Section 3.2. 


\begin{tabular}{|c|c|}
\hline \multicolumn{2}{|c|}{ Sender - 32 bits (IPv4) } \\
\hline $\begin{array}{c}\text { Message Flags - } 16 \\
\text { bits }\end{array}$ & Block Count - 16 bits \\
\hline \multicolumn{2}{|c|}{ Sender $_{1}-32$ bits (IPv4) } \\
\hline \multicolumn{2}{|c|}{ Receiver $_{1}-32$ bits (IPv4) } \\
\hline Block Flags $_{1}-16$ bits & $\begin{array}{c}\text { Indices Pair Count }_{1}- \\
16 \text { bits }\end{array}$ \\
\hline Begin Index ${ }_{11}-16$ bits & End Index $11-16$ bits \\
\hline Begin Index $12-16$ bits & End Index $12-16$ bits \\
\hline$\ldots$ & $\ldots$ \\
\hline Begin Index $x_{1 n}-16$ bits & End Index $1 \mathrm{n}-16$ bits \\
\hline
\end{tabular}

Figure 4: Proposed structure of ACK message.

\section{Evaluation}

\subsection{Analysis}

Here we introduce the fundamental components of the analysis of SERAC's performance bounds; however, a formal proof and complete pursuit of the model analysis is outside the scope of this paper.

\subsubsection{Epidemic Spreading Model}

The Kephart-White model [14] of epidemic propagation in a directed graph defines the following differential equation to find the proportion of a network that is infected.

$$
\frac{d I}{d t}=\beta\langle k\rangle I(1-I)-\delta I
$$

Where $I$ is the fraction of nodes in the network that are infected, $k$ represents the average connectivity of the nodes in the network, $\beta$ is the viral birth rate, and $\delta$ is the viral death rate.

When applying this model to MANETs, Mickens and Noble find that the KW model differs greatly in many cases from simulation results; therefore, the authors propose the use of probabilistic queues to refine the modeling of infections within mobile environments [9]. They model the epidemic spreading of an infection in a mobile network of nodes as a series of queues, each of which represents a given level of node connectivity, $k_{i}$, where $i$ is an integer value between 0 and $N-1$ for a network of $N$ nodes. Then, the $\mathrm{KW}$ model is applied to each queue:

$$
\frac{d I_{Q_{k_{i}}}}{d t}=\beta \cdot k_{i} I_{Q_{k_{i}}}\left(1-I_{Q_{k_{i}}}\right)-\delta \cdot I_{Q_{k_{i}}}
$$


And, to determine the overall infection percentage of the entire network:

$$
\left.I=\sum_{k_{i=0}}^{k_{i=N-1}} \| I_{Q_{k_{i}}}\right\rfloor\left[Q_{k_{i}} \text { length }\right]
$$

\subsubsection{Controlling Birth/Death Rates}

We propose to use Beacon Interval (BI), Retransmit Wait Time (RTW), and Times-to-Send (TTS), as defined in Section 3.1, and adjusting the percent of storage we use as ACK buffer vs. Data buffer to control $\beta$ and $\delta$ in the above models for both the Infection Epidemic (IE) and the Cure Epidemic (CE).

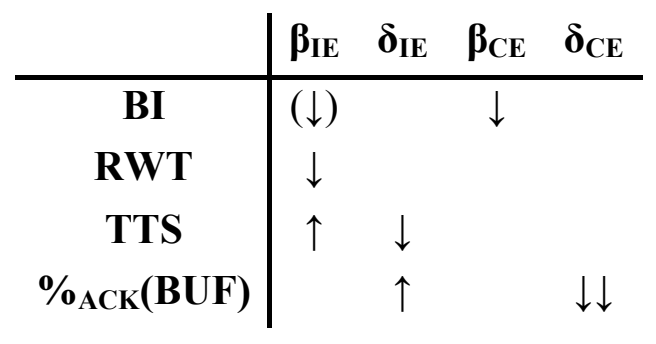

Table 1: An upward pointing arrow indicates that an increase in the control value (BI, RWT, TTS, and $\left.\%_{\mathrm{ACK}}(\mathrm{BUF})\right)$ increases the corresponding rate $(\beta$ or $\delta)$.

In Table 1 we show how increases in the controls $\left(B I, R W T, T T S\right.$, and $\left.\%_{A C K}(B U F)\right)$ will affect the rates $(\beta$ and $\delta$ ) of the KW-based model of Mickens and Nobel for both the IE and the CE. It should be possible to determine both analytically and empirically the correct parameters of the epidemic behaviors (BI, RWT, and TTS) for both infection and cure to optimize the system; however, a formal proof and analysis is not provided here.

\subsection{Simulation}

We have initiated the implementation of simulations to explore the potential performance gains of SERAC. We are using OPNET's Modeler to develop models of MANETs of 10s to 100s of mobile nodes in which we will compare metrics of out-of-the-box (DSR, AODV and OLSR) and custom (pure ER, passive curing in ER and SERAC) protocols. To generalize the models we are using randomly generated point-to-point MANET traffic at a relatively low data rate to emulate delay tolerant traffic and will initially have node mobility as described by the random-waypoint model. The current performance metrics of interest include message end-to-end delay, dropped traffic, overhead (communication and storage), and radio usage (energy consumption). Results are expected to more completely describe the behavior of our proposed scheme. Below we present some preliminary results as an example of the work that is currently on-going.

We loosely define "connectivity" as the ability of a routing protocol to reach all other nodes in the network. The dynamic nature of MANET topologies requires that routing protocols can handle the changing instantaneous connectivity, and we then examine the connectivity over time by simulating a network with point-to-point traffic at very low data rates to avoid stressing throughput limitations. Finally, in order to observe the performance of classical MANET routing protocols as we transition into 
the DTNs, we parametrically reduce radio range (Tx power). The results of these simulations are shown in Figure 5.

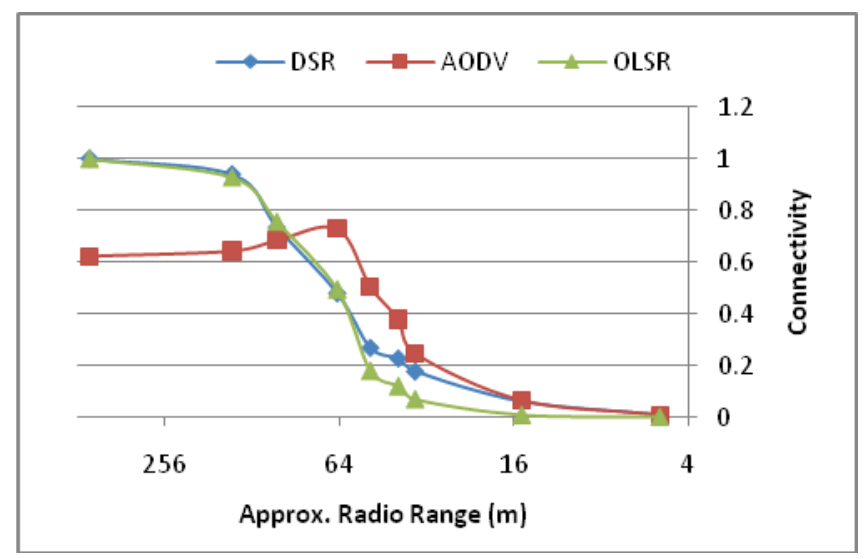

Figure 5: Connectivity for a classical MANET routing protocols breaks down as radio range relative to inter-node distance is reduced.

The plot in Figure 5 shows that, as radio range decreases past the "average" distance between nearestneighbor nodes (simulation was done with 16 nodes in a $200 \mathrm{~m}$ x $200 \mathrm{~m}$ field), the classical MANET routing protocols quickly breakdown. And, at a radio range of 15 meters, all the classical protocols have a connectivity of less than $10 \%$.

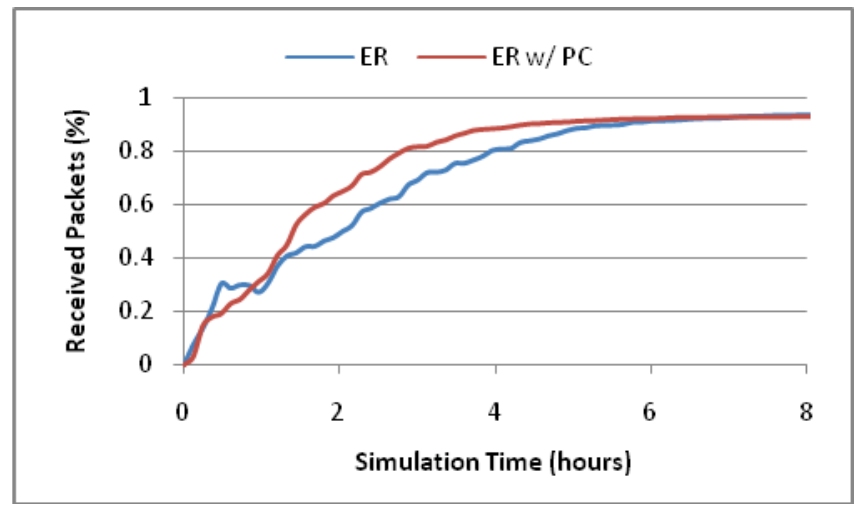

Figure 6: ER-based protocols (at 15m radio range) are able to reach more of the nodes, i.e. have higher connectivity, in opportunistic networks.

In contrast to classical MANET routing protocols the ER-based protocols shown in Figure 6 are able to reach nearly $90 \%$ connectivity at 15 meters over as little as 6 hours. In this simulation the traffic source in each node is active for the first hour of the simulation then stops, in order to allow us to see what percent of total traffic arrives at destination.

The hour reduction in delay that is found simply by using a simple passive cure (PC) is of particular interest. With passive curing the benefit is simply that cured messages are smaller than data messages; however, in SERAC we provide not only a mechanism to reduce the space needed to transfer ACKs 
(cures), but we also prevent unnecessary transmission of data messages, which should greatly reduce the overall consumption of resources and thus improved performance.

\section{Future Work}

In this paper we proposed SERAC, a scheme exploiting existing techniques found in epidemic information dissemination. SERAC utilizes "cure" messages that are designed to propagate at higher rates than original data messages ("infections"). The work is intended to support a variety of opportunistic networking scenarios including inter-vehicle communication, data mules, and monitoring scenarios in which mobile devices share infrequent contact. One might use ER-based routing such as SERAC in a low-power radio mode for delay tolerant traffic and switch to a more conventional routing scheme by increasing radio power for traffic requiring tighter delay bounds.

Although SERAC has not yet been fully implemented and deployed on a test bed, it represents our baseline approach to attacking the opportunistic networking challenge. Moreover, we expect future simulation results that will more completely validate our observations and initial analysis of SERAC behavior.

\section{References}

[1] Amin Vahdat and David Becker, "Epidemic Routing for Partially-Connected Ad Hoc Networks," Technical Report CS-200006, Duke University, April 2000.

[2] Anders Lindgren, Avri Doria, and Olov Schelén, "Probabilistic Routing in Intermittently Connected Networks," Mobile Computing and Communications Review, July 2003.

[3] Natasa Sarafijanovic-Djukic, Michał Piórkowski, and Matthias Grossglauser, "Island Hopping: Efficient Mobility-Assisted Forwarding in Partitioned Networks," IEEE SECON 2006, Reston, VA, September 2006.

[4] Yong Wang, Sushant Jain, Margaret Martonosi, and Kevin Fall, "Erasure-Coding Based Routing for Opportunistic Networks," ACM SIGCOMM Workshop on Delay Tolerant Networks, 2005.

[5] Ling-Jyh Chen, Chen-Hung Yu, Tony Sun, Yung-Chih Chen, and Hao-hua Chu, "A Hybrid Routing Approach for Opportunistic Networks," AMC SIGCOMM Workshop on Challenged Networks, 2006.

[6] Melissa Ho, Stephen Hailes, Vladimir Dyo, Damir Pavušin, and Jasper Weener, "Improving Resource Consumption for Epidemic Routing in Intermittent Networks,"

http://www.melissa.myensim.

net/research/epidemic/epidemic.pdf.

[7] Khaled A. Harras, Kevin C. Almeroth, and Elizabeth M. Belding-Royer, "Delay Tolerant Mobile Networks (DTMNs): Controlled Flooding in Sparse Mobile Networks," In IFIP Networking, 2005.

[8] Patrick T. Eugster, Rachid Guerraoui, Anne-Marie Kermarrec, and Laurent Massoulié, "Epidemic Information Dissemination in Distributed Systems," Computer, Vol. 37, Issue 5, May 2004.

[9] James W. Mickens and Brian D. Noble, "Modeling Epidemic Spreading in Mobile Environments," In Proceedings of the 4th ACM Workshop on Wireless Security, September 2005.

[10] C.E. Perkins and P. Bhagwat, "Highly Dynamic Destination-Sequenced Distance-Vector Routing (DSDV) for Mobile Computers,” in Proc. Sigcomm, 1994. 
[11] D. Johnson and D. Maltz, "Dynamic Source Routing in Ad Hoc Wireless Networks", Mobile Computing, Chapter 5, pages 153-181, Kluwer Academic Publishers, 1996.

[12] Charles E. Perkins and Elizabeth M. Royer, "Ad hoc On-Demand Distance Vector Routing," Proceedings of the 2nd IEEE Workshop on Mobile Computing Systems and Applications, New Orleans, LA, February 1999.

[13] L. Lilien, Z. H. Kamal, and A. Gupta, "Opportunistic Networks: Challenges in Specializing the P2P Paradigm," In Proceedings of the $17^{\text {th }}$ International Conference on Database and Expert Systems Applications, September 2006.

[14] J. Kephart and S. White, "Directed-graph Epidemiological Models of Computer Viruses," In Proceedings of the IEEE Computer Symposium on Research in Security and Privacy, May 1991.

[15] T.D.C. Little and A. Aragwal, "An Information Propagation Scheme for VANETs," In IEEE Intelligent Transportation Systems Conference, Austria, September 2005.

[16] Philo Juang, Hidekazu Oki, Yong Wang, Margaret Martonosi, Li-Shiuan Peh, and Daniel Rubenstein, "Energy-Efficient Computing for Wildlife Tracking: Design Tradeoffs and Early Experiences with ZebraNet," In ASPLOS, October 2002.

[17] Tara Small and Zygmunt J. Haas, "The Shared Wireless Infostation Model - A New Ad Hoc Networking Paradigm (or Where there is a Whale, there is a Way)," In Proceedings IEEE MobiHoc '03, 2003.

[18] Aleksandar Milenkovic, Chris Otto, Emil Jovanov, "Wireless Sensor Networks for Personal Health Monitoring: Issues and an Implementation," Computer Communications (Special issue: Wireless Sensor Networks: Performance, Reliability, Security, and Beyond), Vol. 29, No. 13 14, 2006, pp. 2521-2533.

[19] Alex (Sandy) Pentland, Richard Fletcher, and Amir Hasson, "DakNet: Rethinking Connectivity in Developing Nations," Computer, vol. 37, no. 1, January 2004.

[20] A. Agarwal and T.D.C. Little, "Prospects for Networked Vehicles of the Future," Proc. Workshop on Smart Transportation, 13th IEEE Real-Time and Embedded Technology and Applications Symposium, Bellevue, WA, April 2007. 